\title{
Maser Astrometry with VLBI and Galactic structure
}

\author{
Mareki Honma* \\ Mizusawa VLBI Observatory, National Astronomical Observatory of Japan \\ SOKENDA (The Graduate University for Advanced Studies) \\ E-mail: mareki.honma@nao.ac.jp
}

In this paper we present the current status of VLBI maser astrometry and discuss the Galaxy structure revealed with the maser astrometry. To data parallaxes and proper motions have been obtained for more than 100 sources with EVN, VERA and VLBA, and the fundamental Galactic structure has been explored based on these astrometric measurements. The Galactic constants are already determined at a few $\%$ level accuracy, with most-likely values with $R_{0} \approx 8.3 \mathrm{kpc}$ and $\Theta_{0} \approx 240 \mathrm{~km} \mathrm{~s}^{-1}$. The latter suggests that the Galaxy's rotation velocity is higher than the IAU standard of $\Theta_{0}=220 \mathrm{~km} \mathrm{~s}^{-1}$, indicating that the existence of more dark matter than previously expected. Moreover, maser astrometry results provide constraints on basic structure of the spiral arm, including their locations, orientations and dynamics. We also discuss the future prospect of VLBI maser astrometry in 2020's, and propose that a globally combined array comprising $\sim 7 \%$ of SKA's collecting area would significantly boost up the science capability of VLBI including maser astrometry.

12th European VLBI Network Symposium and Users Meeting,

7-10 October 2014

Cagliari, Italy

\footnotetext{
* Speaker.
} 


\section{Preface: historical connection between Cagliari and Mizusawa observatory}

Both Cagliari observatory and Mizusawa observatory have the common origin in the history of astronomy. They were founded in 1899 as ones of the six observatories of the International Latitude Service (ILS). The six observatories were: Carloforte in Italy (located on San Pietro Island next to Sardinia), Tscharudjui in Turkestan, Mizusawa in Japan, Ukiah (California), Cincinnati (Ohio) and Gaithersberg (Maryland) in US, all of which were located at a common northern latitude of $39^{\circ} 08^{\prime}$. They were built for conducting optical observations of stellar transits with an aim to trace the variation of the Earth's polar motion. At the end of 19th century, the ILS was the most modern and big international project in natural science under the world-wide collaboration.

Immediately after the start of the ILS observations, Mizusawa observatory made a significant contribution to the ILS: Dr. Hisashi Kimura, the first director of the ILS Mizusawa observatory discovered the so-called " $z$-term" [6], which was an additional term necessary to better explain the observed latitude variation, i.e.,

$$
\Delta \phi=x \cos \lambda+y \sin \lambda+z
$$

Here $\Delta \phi$ is the latitude variation, a vector $(x, y)$ describes the instantaneous positon of the north pole, and $\lambda$ is the observatory longitude, respectively. The interpretation of the $z$-term was given $\sim 70$ yr later than Kimura's discovery when Wako[19 revealed that this term reflects the uncorrected nutation originating from the liquid core of the Earth.

The ILS observations have been continued around 1980, and then both the Mizusawa and the Cagliari observatory shifted its focus toward geodetic and geodynamical studies, and lately, at the beginning of the third millennium, also toward radio astronomy and VLBI, as in 1980's the VLBI technology was already available for precisely measuring station positions as well as Earth's rotation. The ILS Mizusawa observatory has been reformed as Mizusawa VLBI observatory as a part of NAOJ (National Astronomical Observatory of Japan), and now it operates VERA (VLBI Exploration of Radio Astrometry) as its major facility. The ILS Carloforte observatory has become
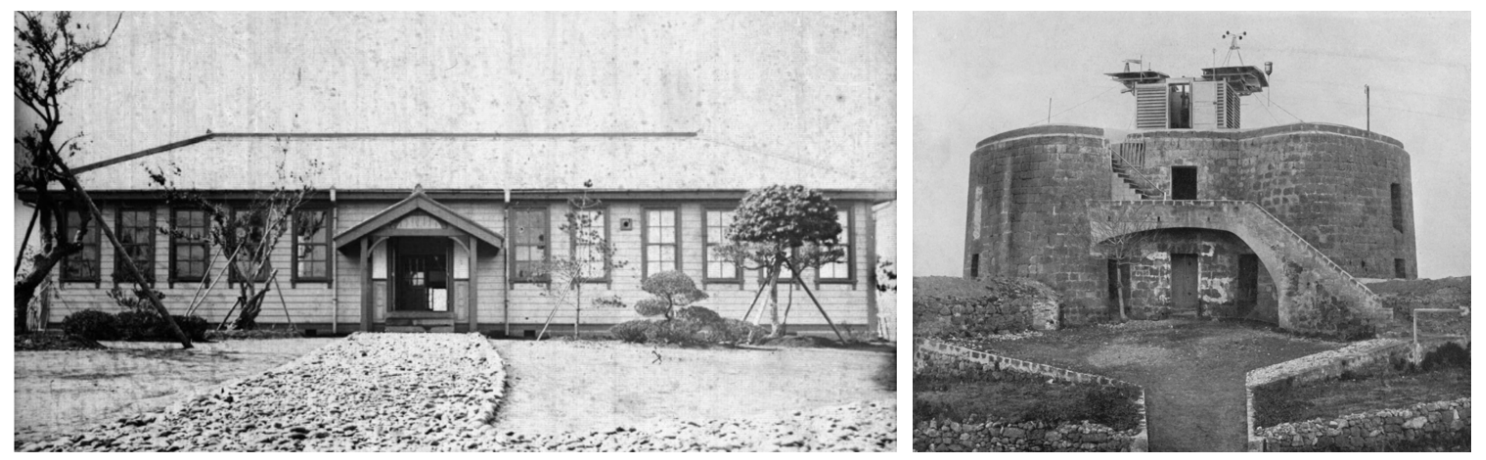

Figure 1: Left: ILS Mizusawa station, the predecessor of the Mizusawa VLBI observatory of NAOJ. Right: ILS Carloforte station in San Pietro island, the predecessor of the Cagliari Observatory of INAF. 
a part of Cagliari Astronomical Observatory of INAF, which is now operating the Sardinia Radio Telescope (SRT) project. Nowadays Japanese and Italian radio astronomers are conducting test VLBI observations between these countries with the participation of the SRT, and hence these two observatories are still actively collaborating after a long history over $100 \mathrm{yrs}$ !

\section{Astrometry with VLBI}

VLBI (Very Long Baseline Interferometry) provides the highest angular resolution amongst existing telescopes at any wavelength of electromagnetic wave. With such a high angular resolution, VLBI can be used either to obtain super-fine images of celestial objects with the highest resolution, or to precisely measure the positions of celestial objects, i.e., high-accuracy astrometry. For the latter, recent progress in phase-referencing technique for compensating the troposphere has revolutionized astrometry accuracy, and now astrometry with $\mu$ as level accuracy is possible (for review, see [12]).

Astrometric accuracy in phase-referencing VLBI can be determined as

$$
\Delta \theta \approx \theta_{\mathrm{SA}} \frac{c \Delta t}{B} .
$$

Here $\theta_{\mathrm{SA}}$ is the separation angle between the target source and the reference source, $c$ is the speed of light, $\Delta t$ is the measurement error in delay (usually limited by the water vapor fluctuation in the troposphere), and $B$ is the maximum baseline length, respectively. Note that in the above equation both $\Delta \theta$ and $\theta_{\mathrm{SA}}$ are described in radian. If one has a baseline $B$ of $8000 \mathrm{~km}, \theta_{\mathrm{SA}}$ of $1 \mathrm{deg}(\sim 1 / 57$ radian), and $c \Delta t$ of $1 \mathrm{~cm}$, one can achieve $\Delta \theta$ of $\sim 5 \mu$ as !

Currently this accuracy is unprecedented by optical astrometry such as HIPPARCOS ([9]), and GAIA mission is on its way to reach down at the same accuracy level with VLBI, with the final catalog expected to be released in early 2020's. VLBI already achieved a distance measurement of sources at 5-10 kpc (i.e., [12]), and thus Galactic-scale astrometry is readily being conducted.

Currently there are three major VLBI arrays in the northern hemisphere regularly conducting astrometry: EVN (European VLBI Network) in Europe, VERA in Japan, and VLBA (Very Long Baseline Array) in US. Also in the southern hemisphere, LBA (Long Baseline Array) in Australia is the only VLBI array in operation, and recently there has been Galactic-scale astrometry conducted by LBA ([7]).

For doing VLBI astrometry, one requires sources with high brightness temperature, and the most commonly observed sources are maser sources such as $\mathrm{OH}, \mathrm{H}_{2} \mathrm{O}$ and $\mathrm{SiO}$ masers associated with star forming regions and/or later type stars. In addition to maser sources, there are other types of sources which are also interesting targets for VLBI astrometry, such as the Galaxy center black hole Sgr A*, micro-QSOs, pulsars and so on [12].

\section{Recent results in maser astrometry}

Since early 2000's, VLBI maser astrometry at kpc-scale has been routinely conducted with VLBA, VERA and EVN. As one of pioneering results, Xu et al. [20] and Hachisuka et al. [2] reported VLBA observations of $\mathrm{W} 3 \mathrm{OH}$ with a parallax distance of $1.95-2.05 \mathrm{kpc}$ at a $2-3 \%$ accuracy. For VERA, Honma et al. [4] obtained S269's distance to be $5.28 \mathrm{kpc}$ at $4 \%$ level. Rygl et al. 


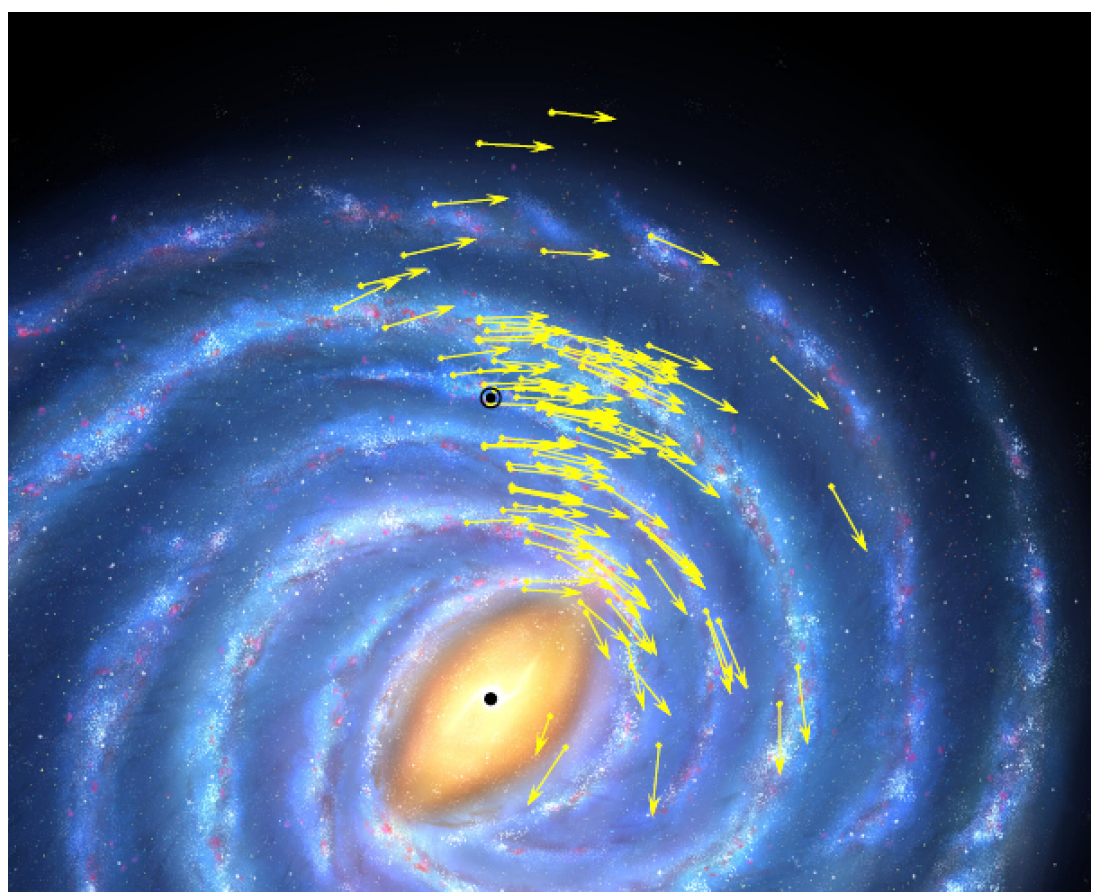

Figure 2: Distribution of 114 maser sources observed with EVN, VERA and VLBA superposed on the artistic view of the Milky Way Galaxy. The dots with arrows show the maser sources, with vectors showing their proper motions. The black spot is the Galaxy center, and the black spot with a circle is the Sun.

[14 15] also reported maser parallax measurements with EVN, demonstrating that EVN is also a powerful array for conducting Galactic-scale astrometry.

Following the successful initial investigations above, these arrays have been involved in maser astrometry on regular basis: VERA has been continuing the operation as a dedicated array to VLBI astrometry, and VLBA has been conducting kpc-scale maser astrometry as BeSSeL project. Also there have been continuing efforts on VLBI astrometry using EVN. Thanks to these activities, currently (as of the end of 2014), in total accurate parallaxes and proper motions have been obtained for 114 Galactic maser sources associated with the Galactic star-forming regions. Figure 2 shows the source distribution on a plan view of the Galaxy, with an artistic impression of the Milky Way's disk and spiral arms. As seen in the figure, the maser sources distribute at distances from a few $100 \mathrm{pc}$ to $10 \mathrm{kpc}$ from the Sun, and they cover a large fraction of the Galactic disk, being useful for tracing the Galactic structures such as spiral arms and the central bar. Note that the source distribution is still biased toward the northern part of the Galaxy due to the geographic locations of the VLBI arrays, and thus the observations of the southern maser sources remain important issues for the near future. Also important is to obtain the parallax results beyond $10 \mathrm{kpc}$, for which one may need better array with longer baselines and/or better calibration accuracy.

Also prominent in figure 2 is the Galactic rotation (shown as vectors), which appears as a circular rotation in the first order approximation. The acceleration due to the circular rotation is clearly seen in the difference of the directions of source motions, and based on that one can determine the position of the dynamical center of the Galaxy as well as the mean speed of the 
Table 1. Galactic constant determinations based on VLBI astrometry.

\begin{tabular}{cccc}
\hline & $\mathrm{N}_{\text {src }}$ & $R_{0}$ & $\Theta_{0}$ \\
\hline Reid+(2009) & 18 & $8.4 \pm 0.6 \mathrm{kpc}$ & $247 \pm 16 \mathrm{~km} / \mathrm{s}$ \\
Honma+(2012) & 52 & $8.05 \pm 0.45 \mathrm{kpc}$ & $238 \pm 14 \mathrm{~km} / \mathrm{s}$ \\
Reid+(2014) & 103 & $8.34 \pm 0.16 \mathrm{kpc}$ & $240 \pm 8 \mathrm{~km} / \mathrm{s}$ \\
\hline
\end{tabular}

Galactic rotation (i.e., the Galactic constants $R_{0}$ and $\Theta_{0}$ ). Based on the detailed statistical analyses, initial results on the Galactic constants are reported by Reid et al. [11] using 18 sources, resulting in $R_{0}=8.4 \pm 0.6 \mathrm{kpc}$ and $\Theta_{0}=247 \pm 16 \mathrm{~km} \mathrm{~s}^{-1}$. Later Honma et al. [5] refined the Galactic constants using 52 sources available in 2012, obtaining $R_{0}=8.05 \pm 0.45 \mathrm{kpc}$ and $\Theta_{0}=238 \pm 14 \mathrm{~km} \mathrm{~s}^{-1}$, respectively. Most recently Reid et al. [13] compiled 103 sources to obtain $R_{0}=8.34 \pm 0.16 \mathrm{kpc}$ and $\Theta_{0}=240 \pm 8 \mathrm{~km} \mathrm{~s}^{-1}$. These results are consistent with each other within the error bars, and the last one already reached at 2-3\% accuracy in Galactic constant determination, demonstrating the power of the VLBI astrometry (see table 1). Also important is that the Galactic rotation $\Theta_{0}$ seems larger than the IAU standard of $220 \mathrm{~km} \mathrm{~s}^{-1}$, indicating that the mass of the Galaxy is larger than previously expected. This implies that there exists more dark matter in the Galaxy, which brings a strong impact on the dark matter particle detection experiments on-going on the Earth. In addition to the Galactic constants, one can also obtain the rotation curve shape and mean peculiar motions of the sources, which are also important for understanding the Galaxy's structure.

Beyond the circular rotation, the maser astrometry provides valuable information on the detailed structure on the Galaxy. The spiral arm structure is the main target of such studies: Reid et al.[13] traced the spiral structure with the maser distributions and determined the pitch angles of the spiral arms such as Sagittarius, Perseus and Outer arms. Also, Choi et al.([1]) attempted to trace the source distribution in the Perseus arms and they used it to revise the kinematic distance to these sources. Most recently, Sakai et al. ([16]) tried to explain the non-circular motion along the Perseus arm with a density-wave-type dynamical model developed by Piñol-Ferrer et al. ([10]), and revealed that non-circular motions associated with Perseus arm sources could be described by the density-wave model with a set of reasonable parameters.

Beside the Galactic structure, late-type stars such as super-giant stars and AGB stars are interesting targets for maser astrometry with VLBI. Pioneering works have been done with VLBA by van Langevelde et al. ([17]) and Vlemmings et al. ([18]), showing that these maser sources are also good targets for VLBI astrometry, though these sources are somewhat closer to the Sun (a typical distance of $\mathrm{kpc}$ or less). Yet astrometry of these sources provide valuable information on stellar mass-loss, structure of circum-stellar envelope, and period-luminosity relation for the pulsating stars. Recently Nakagawa et al.([8]) presented a period-luminosity relation for Miras based on VLBI astrometry, which will be an important basis for calibrating the distances to other AGB stars.

\section{Future prospect}

Currently in East Asia, VERA and KVN (Korean VLBI Network) are in joint operation on 


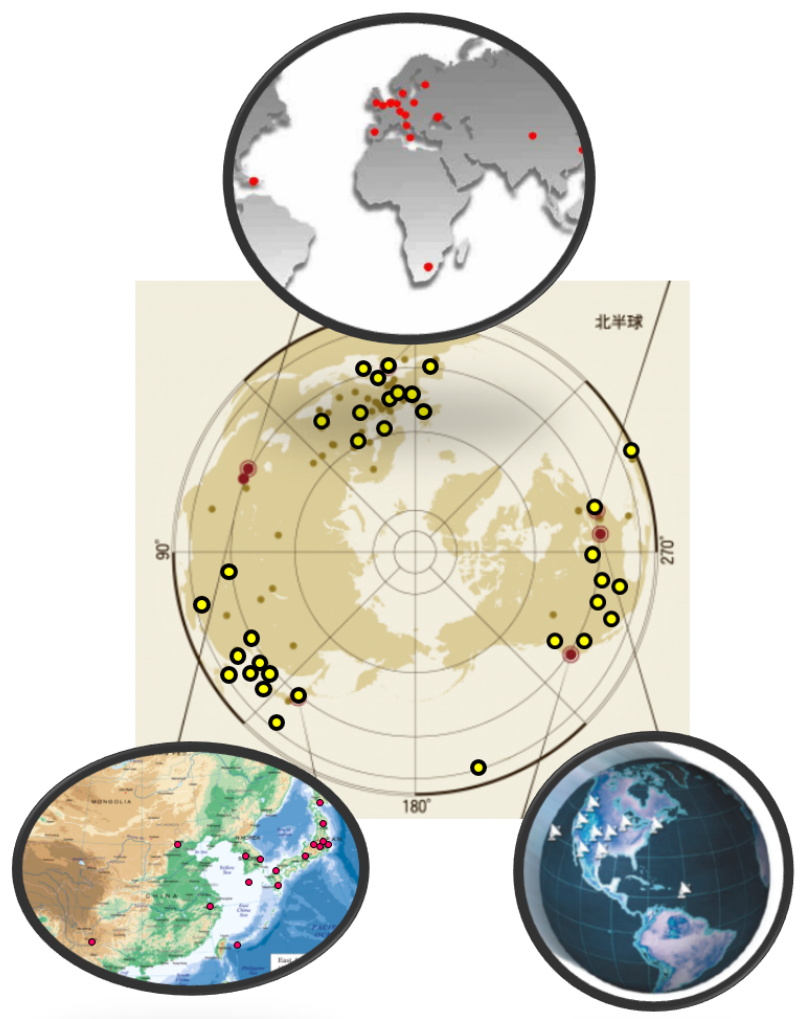

Figure 3: Major VLBI Arrays in northern hemisphere. Clockwise from top, EVN, VLBA and EAV. Combining these arrays would make a global VLBI network having a collecting area of $\sim 7 \%$ of SKA.

regular basis as KaVA array (KVN and VERA Array), consisting of seven of 20m-class antennas. In addition, there is a plan to establish EAVN (East Asian VLBI Network) by combining stations in Japan, Korea and China. For doing this, SHAO $25 \mathrm{~m}$ and the new $65 \mathrm{~m}$ telescopes (and others as well) in Chinese VLBI Network are possible stations contributing to EAVN from China. With these possible extensions, we will continue operation for next 10 years, and hopefully combination of VERA, VLBA/BeSSeL and EVN data (and also GAIA, the European space astrometry mission) will be of great help for understanding the details of the Galactic structures.

In early 2020's, GAIA will also complete astrometric measurements for billions of stars in the Galaxy. Since GAIA observes stars and VLBI observes maser sources (mostly SFR, which traces gaseous components in the Galaxy), these two are complementary to each other, and thus both of the optical and radio astrometry are indeed important for the understanding the Galaxy dynamics: while the stars are collisionless system, the SFRs (gaseous components) are collisional, and hence these two populations should show totally different motions although they are in the same Galactic potential. Therefore, for testing dynamical models of the Galaxy, the information of the both populations are required. In this sense, Galactic astrometry in 2020's will be exciting with a combination of GAIA and VLBI astrometry.

For further future, more global collaborations should be essential, and the combination of the more stations should be considered to realize better sensitivity, better angular resolution and better astrometry. Thus, a combined array of EVN, VERA and VLBA (and also more stations even in the southern hemisphere) is something we have to consider for our future (e.g., figure 3). The 
combined array would have a collecting area corresponding to $~ 7 \%$ of SKA, and so part of the science for SKA phase-I may be done with such a combined global array, significantly boosting the science capability of VLBI including high-accuracy astrometry.

Regarding astrometry, the large collecting area (+ recording with a wider bandwidth) allows us to observe fainter sources such as those in the far side of the Galaxy. Also, higher sensitivity enables us to conduct astrometry using a nearby calibrator, which will contribute in obtaining better astrometry accuracy. If one assumes that there is a sensitivity gain of an order of magnitude, then the average separation between the target and reference should be reduced by a factor of $10^{3 / 4} \approx 5.6$ (assuming the isotropic reference distributions), resulting in astrometry accuracy better by a factor of $\sim 5$. Therefore, if such a powerful array would indeed become available, the whole Galaxy could be in the reach of VLBI astrometry.

\section{References}

[1] Choi Y. K., et al., 2014,, ApJ, 790, 99

[2] Hachisuka et al. 2006, ApJ, 645, 337

[3] Hachisuka et al. 2015, ApJ, 800, 2

[4] Honma M., et al., 2007, PASJ, 59, 889

[5] Honma M., et al., 2012, PASJ, 64, 136

[6] Kimura H., 1902, AJ, 27, 107

[7] Krishnan V., et al., 2015, ApJ, submitted

[8] Nakagawa A., et al., 2014, PASJ, 66, 101

[9] Perryman M. Am C. et al., 1997, A\&A, 323 L49

[10] Piñol-Ferrer, N., Lindblad, P. O., \& Fathi, K., 2012, MNRAS, 421, 1089

[11] Reid M. J. et al., 2009, ApJ, 700, 137

[12] Reid M. J. \& Homa M., 2014, ARA\&A, 52, 339

[13] Reid M. J. et al., 2014, ApJ, 783, 130

[14] Rygl K., et al., 2010, A\&A, 511, 2

[15] Rygl K., et al., 2010, A\&A, 539, 79

[16] Sakai N., et al., 2015, PASJ, submitted

[17] van Langevelde H. J., et al., 2000, A\&A, 357, 945

[18] Vlemmings W., et al., 2003, A\&A, 407, 213

[19] Wako Y., 1970, PASJ, 22, 525

[20] Xu Y, et al., Science, 311, 54 